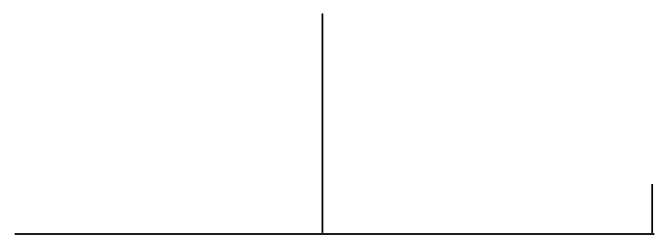

Rev. Latinoam. Psicopat. Fund., São Paulo, v. 13, n. 4, p. 599-616, dezembro 2010

\title{
L'alexie aujourd'hui. Quelques hypothèses sur un symptôme de notre temps*
}

\author{
Tatiana Pellion
}

La difficulté pour un sujet à lire - voire à écrire - est un symptôme actuel de notre civilisation. Plusieurs exemples cliniques viennent interroger le rapport entre apprentissage et savoir inconscient pour un sujet. L'origine de l'envie d'apprendre dans les pulsions freudiennes est soulignée ainsi que la nécessaire fonction de perte des objets petit a (et particulièrement le regard et la voix). Du côté du réel, la fonction des lettres qui se perdent est aussi mise en question. La direction de la cure et la prise en compte de sa temporalité est traitée en vue d'une lecture de l'inconscient comme un jeu de mots, de lettres.

Mots clés: Savoir, corps, pulsion, objet petit $a$

* Cet article est la réecriture d'une intervention aux journées d'études d'Espace Analytique "Comment s'écrit l'inconscient?", dans le cadre d'un atelier intitulé "Les symptômes actuels sont-ils déchiffrables?", le 16 novembre 2008. 
En relisant ta lettre je m'aperçois que l'orthographe et toi, ça fait deux. Serge Gainsbourg (1961). "En relisant ta lettre", p. 47.

Il n'y a que la poésie qui permette l'interprétation. Jacques Lacan (1977). Séminaire "L'insu que sait de l'une bévue s'aile à moure”.

Y-a t'il une évolution des symptômes chez les sujets depuis l'invention de la psychanalyse, il y a plus d'un siècle? Peut-on parler à notre époque de "symptômes actuels"? Et ces symptômes sont-ils déchiffrables? Les sujets qui consultent un psychanalyste viennent au premier entretien - pour beaucoup - se plaindre d'un mal-être (dépression, morosité, difficulté à rencontrer un partenaire amoureux, mal de dos, migraines, troubles digestifs, difficultés à dormir, troubles du comportement alimentaire etc.); ils ont souvent tenté auparavant d'apaiser leurs corps avec des séances d'ostéopathie, de sophrologie, de kinésithérapie, ou bien de prendre des médicaments pour dormir, des anxiolytiques, ou encore des antidépresseurs. Certains se sont essayés à l'hypnose. D'autres font du yoga. Mais, constatent-ils: quelque chose insiste. Ce "quelque chose" - à quoi nous avons à faire dès les premières séances - nous commençons par le recevoir, sans savoir de quoi il s'agit. Nous l'avons appris de notre analyse. Ne pas savoir à priori, faire silence afin d'ouvrir un espace de parole au sujet, écouter la demande, circonscrire petit à petit cette "chose" afin que la demande se formule un peu autrement. Notre propre analyse nous a appris l'importance de la première rencontre avec un analyste, des premiers entretiens. Et d'ailleurs, si le temps du déchiffrage est nécessairement long, on ne se souvient dans l'après-coup que de quelques séances, quelques moments de comprendre qui vont petit à petit s'inscrire et permettre une bascule de la position subjective. On perd beaucoup du temps pendant une analyse. Son trajet est souvent jalonné de temps d'arrêts, de suspensions; et ces temps sont nécessaires, ils participent à la construction d'une certaine ponctuation, nous y reviendrons un peu plus loin. 
"Je viens ici, mais je veux que ça aille vite. Je ne veux pas en avoir pour dix ans" nous dit une jeune femme ayant pris rendez-vous dans l'urgence en plein mois d'août, après un épisode de sciatique aïgue l'ayant clouée au lit pendant près d'un mois. Et pourtant, elle vient, elle est là, mais que demande t-elle? Il nous semble que les demandes des sujets - sujets en état d'urgence, sujet en mal-être - se multiplient actuellement sous couvert de ces divers symptômes que nous avons évoqué. Nous voilà donc, avec ces sujets là, particulièrement confrontés à un certain rapport au temps. Aller vite, qu'est ce que cela veut-dire pour un sujet? Et surtout, aller vite pour aller où, dans quel lieu - question nécessairement topologique.

\section{Une culture du voir}

Que dirait Freud de notre civilisation actuelle, lui qui dans son Malaise dans la culture, en 1929, remarquait avec pessimisme que l'invention d'un certains nombre d'outils tels que le téléphone ou l'appareil photo permettait à l'individu d'attendre de moins en moins, de réduire le manque. Le développement d'un certain nombre de techniques a repoussé plus loin les limites de la performance qui, selon Freud, étaient pour l'homme autrefois inatteignables, puisque attribuées aux dieux. ${ }^{1}$ Il a ainsi permis d'atteindre une certaine "perfection" des organes sensoriels - notamment de l'oeil et de l'ouïe - jusqu'à "accomplir tous les souhaits de contes". Ces progrès ont signés une certaine omnipotence de l'homme, qui est devenu, à l'aide de ses "organes adjuvants", une sorte de dieu grandiose, pouvant satisfaire un certain nombre d'ideaux.

Ces organes adjuvants font à l'heure actuelle corps avec le sujet. Que dirait Freud de cet ère du numérique, de la photocopieuse, des textes, de la télévision et d'internet, de cette civilisation où les images sont prégnantes, où la technique et le savoir scientifique se manifestent par une certaine toute puissance du regard sur l'être humain?' Comme le souligne Alain Didier-Weill, la technique scientifique avec ses sondes et ses machines, va actuellement jusqu'à construire une sorte de

1. Ainsi, les lunettes permettent de corriger "les défauts de la lentille de son oeil", le télescope de "voir à des distances lointaines", le microscope de surmonter "la frontière de la visibilité"; enfin, avec l'appareil photographique, "il a crée un instrument qui retient les impressions visuelles fugitives". Freud, S (1929). Le malaise dans la culture, p. 34.

2. Aujourd'hui, par exemple, quand un enfant ou un adolescent a un exposé à préparer, il peut double cliquer sur Wikipédia et sans soucis recopier le contenu du texte qui s'affiche sur l'écran. 


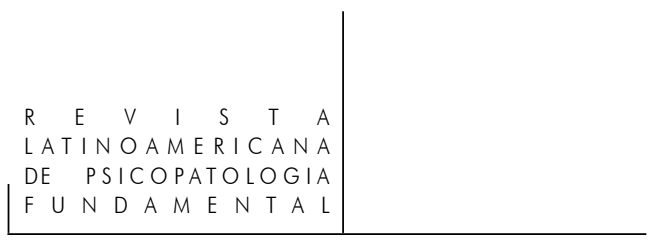

savoir "regardant", jusqu' à vouloir visualiser la rencontre du spermatozoïde et de l'ovule, défiant ainsi l'inconnu, l'inconnaissable, l'inexpliqué de notre venue au monde. Pour Alain Didier-Weill (2003), “ce qui spécifie le malaise de notre monde est lié au fait que le développement scientifique des techniques tend à menacer, de façon nouvelle, l'humanisation promise par la vie de la parole". La responsabilité de l'analyste - de même que celle de l'artiste - est donc, comme il le souligne, de lutter d'une certaine manière contre cette menace, "contre ce qui attente à l'existence de la parole" (p. 123).

Nous avons appris du travail avec les adolescents combien cet "aller vite" - que nous évoquions plus haut - vient interroger le rapport du sujet à la pulsion (pulsion orale, pulsion invoquante) et sans doute à la trace. Car aller vite, c'est souvent ne pas laisser de traces. L'adolescent a la plupart du temps des difficultés avec l'oral - la parole et l'alimentation. Soit il parle peu, soit il parle à toute vitesse. Comme le remarque Jean Bergès, "il ne parle pas, il crie, il parle de telle façon qu' on ne comprend rien; il part du principe que vous devinez et que vous ne comprenez rien à ce qu'il pense mais que vous faites celui qui ne comprend pas parce qu'en réalité vous devinez". Comme si on pouvait lire en lui; comme si il était d'une certaine manière assez transparent pour son interlocuteur. Et cette transparence - poursuit Bergès - n'est pas sans liens avec "les traces que laissent l'écriture". Or l'adolescence est justement le moment où les écritures se remanient pour un sujet. ${ }^{3}$

\section{Des sujets en alexie}

Si nous partons de l'hypothèse de Lacan (1972-73) dans son séminaire Encore que le symptôme c'est "ce qui ne cesse pas de s'écrire” (p. 86), il nous semble intéressant d'envisager la difficulté pour un sujet voire son impossibilité à lire - et donc à écrire - comme un symptôme de notre temps. Chez les enfants

3. Il s'agit en effet pour l'adolescent ou l'adolescente d'assumer sa place par rapport à la sexuation: être garçon ou fille, question signifiante. Il se confronte ainsi à la particularité de l'écriture logique de la sexuation (qui peut, comme l'a développé Jacques Lacan dans son séminaire Encore, s'écrire sous forme de mathèmes). Lacan insiste notamment sur ceci qu'il n'y a pas plus d'adéquation possible entre l'objet et la pulsion qu'entre le sujet et l'objet, ce que (re)découvre le sujet, maintenant pubère, à l'adolescence. Lacan, J. (1972-73). Le séminaire. Livre XX. Encore, p. 173. Bergès, J. (1997). "La dyade, ça n'existe pas". In: Le corps dans la neurologie et dans la psychanalyse, p. 133. 
et les adolescents que nous rencontrons en consultation au CMP de secteur du service de Psychologie et Psychiatrie de l'Enfant et de l'Adolescent du Centre Hospitalier Sainte-Anne, ${ }^{4}$ on appelle cette impossibilité "troubles des apprentissages". Ce terme, n'est plus seulement employé par les pédopsychiatres ou les psychanalystes, mais aussi par les pédiatres, les instituteurs, les professeurs, les conseillers d'orientations, les proviseurs et les équipes pédagogiques. Ce symptôme - véritable phénomène social - est souvent renvoyé du côté de la maladie et de ce qui concernerait la médecine. Alors, comme le développent Jean et Marika Bergès et Sandrine Jean-Calmettes (2002) dans leur travail de recherche: "Que nous apprennent les enfants qui n'apprennent pas?", on propose toute une batterie d'évaluation des capacités cognitives de l'enfant (en tant qu'elles seraient défaillantes), de son rapport à la connaissance, de sa mémoire, de sa concentration, de ses capacités logiques, de compréhension, bref de son intelligence. Ces sujets en difficultés d'apprentissage viennent en consultation adressés par leur école, leur collège, leur pédiatre, ou d'autres encore, ou bien sur inquiétude de leurs parents qui formulent les difficultés de leur enfant le plus souvent ainsi: "il n'arrive pas à se concentrer à l'école!" Qu'estce que cette difficulté de concentration recouvre?

Nous avons choisi ici, en reprenant un terme à la neurologie, d'appeler plus largement cette difficulté à lire des sujets: alexie. Le terme alexie vient du grec $a$ privatif, exprimant la négation, et lexis, le "mot". Il décrit un désordre pathologique de la sphère sensorielle se manifestant principalement par un trouble d'accès à la lecture. L'alexie consiste en la perte des compétences cognitives permettant non seulement la lecture mais également la compréhension du langage écrit et la transposition phonatoire. Le sujet est alors en incapacité à décoder ou à transposer à haute voix le message linguistique écrit. La neurologie le lie à une dysfonction cérébrale. ${ }^{5}$ Nous n'entrerons pas ici dans une distinction détaillée des différentes formes d'alexie au sens de la neurologie: textuelle, avec agraphie, sans agraphie, littérale, verbale. Nous avançons pour notre part l'hypothèse que l'alexie - si elle est dans certains cas d'origine organique - nous concerne aussi en tant qu'elle est un symptôme actuel. Nous allons essayer de l'aborder au travers d'exemples cliniques.

4. 6 ème intersecteur de Psychologie et Psychiatrie de l'Enfant et de l'Adolescent (Centre Médico-Psychologique secteur du 14ème arrondissement). Centre Hospitalier Sainte-Anne. 1, rue Cabanis. 75014 Paris. France.

5. Nous nous référons sur ce point à Bérubé, L. (1991) Traité de Terminologie de neuropsychologie et de neurologie du comportement, p. 65. 


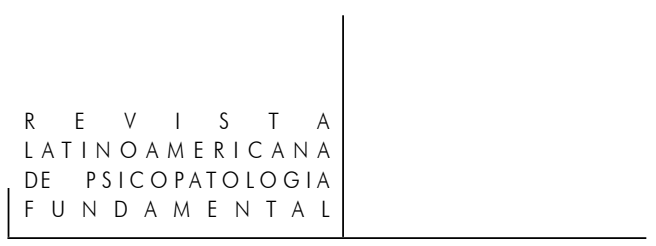

\section{Maureen}

Maureen, belle jeune femme d'une trentaine d'années vient consulter à notre cabinet car elle souffre d'un "mal au cou" que quatres mois de séances d'hypnose n'ont fait cesser. Cela fait plusieurs mois que cela dure et la jeune femme est obligée régulièrement de s'arrêter de travailler. La douleur la prend subitement, la dernière fois nous dit-elle, au beau milieu d'une rame de métro bondé et elle sent que "cela se bloque" en elle. Maureen vit seule depuis quelques années, elle a des liaisons avec des amants de différentes nationalités, la plupart originaires d'Afrique, qui peuvent durer quelque temps, sans qu'aucun projet commun ne puisse se dégager. Ces liaisons se terminent souvent de la même manière: le jeune homme lui demande de lui prêter de l'argent et Maureen se plaint de son inconsistance. Elle met beaucoup d'energie, parfois sans succès, à travailler dans les quartiers difficiles d'une banlieue parisienne afin d'essayer de mettre en place des projets de restauration et de construction des lieux de vie de la cité. Elle entretient une vie sociale assez riche, et une relation avec sa mère d'une grande proximité - "avec ma mère, pas besoin de parler, on se comprend!". Quant à son père, ingénieur de haut niveau, elle n'a que peu de dialogue avec lui. Au premier entretien, Maureen nous confia que la seule chose qui importait pour elle était de partir. Nous lui demandons: "Pour aller où?" Partir à l'étranger, se faire muter car elle constatait que ses symptômes ne se manifestaient pas lors de ses voyages qu'elle aimait effectuer, plusieurs fois par an.

Le travail qu'elle fit, au cours des quatre premières années de son analyse, où ses douleurs survenaient par périodes de manière assez aïgue, la mena à associer sur le "coût" d'une donation que sa mère lui attribua, à l'âge de 25 ans. Il s'agissait d'une somme importante provenant de l'héritage de sa grand-mère maternelle, décédée 6 ans auparavant. Cet héritage, destiné à sa mère, lui avait ainsi été transmis par donation, car cette dernière lui disait qu'elle n'en avait pas besoin. Elle prétendait lui donner cette somme à la condition que Maureen n'en fasse rien d'autre que de s'acheter un appartement. Elle lui avait dit à l'époque avoir fait mettre une clause en ce sens sur le contrat notarié: elle donnait cette somme à sa fille mais gardait toujours "un droit de regard" sur celle-ci, "une procuration" sur cette somme et le bien acquis. La jeune femme semblait croire les dires de sa mère, qui énonçait là pourtant une loi pour le moins étrange.

Une intervention de notre part, demandant simplement à la patiente si elle avait lu l'acte notarié, eut une fonction surprenante. Elle constata en effet qu'elle ne l'avait eu entre les mains que pour le signer, mais qu'elle ne l'avait pas lu, se conformant à l'époque au discours maternel et qu'elle ne savait pas au fond, si cette clause existait ou non. Elle contacta le notaire afin de lui demander un double du contrat, qui ne lui avait jamais été envoyé, et se souvint que cet argent avait 
été gagné par ses grands-parents maternels dans une entreprise de production de céréales, en Afrique. La lecture nouvelle de ce souvenir oublié, la mena à exprimer un certain affect de honte quant à la manière dont cet argent avait été gagné, par la voie du colonialisme de l'époque. Ainsi le "coût" de cette donation s'était déposé toutes ces années sur le corps, produisant des douleurs sur l'organe, qui disparurent, après lecture du symptôme.

Bianca

Prenons un deuxième exemple clinique. Bianca, jeune de 16 ans, vient consulter au CMP depuis l'âge de 12 ans, car elle souffrait au départ de cauchemars et de terreurs nocturnes. Elle avait peur qu'un "voleur" s'introduise dans sa maison et tue sa famille. Cette angoisse la menait à refuser de dormir seule et à aller rejoindre sa mère dans son lit. Bianca vivait avec sa mère, et son frère aîné, Arthur. En 6e à l'époque, elle s'avérait en difficultés scolaires, obtenait des résultats médiocres, avec l'idée de "ne pas être à la hauteur". Ses parents étaient séparés depuis qu'elle avait 3 ans et elle voyait peu son père, ancien gérant d'un théâtre, en arrêt maladie pour une pathologie épileptique associée à des difficultés avec l'alcool. Les premières années du travail psychique que fit Bianca lui permirent d'évoquer son angoisse par rapport à la fragilité de ce dernier - qui pouvait disparaître pendant quelques semaines pour faire des cures de désintoxication, sans qu'elle sache où il se trouvait. Monsieur habitait à l'époque avec sa propre mère et Bianca n'aimait pas se rendre dans cet appartement qu'elle trouvait triste et délabré, à l'image de ce qu'elle se représentait de son père. Quelques entretiens avec lui permirent à Bianca de l'entendre parler de sa rencontre avec sa mère. Sorties jusqu'au petit matin, fêtes avec quelques grands poètes et chanteurs français de l'époque, casinos, dettes de jeux ponctuaient celle-ci, jusqu'à devenir un empêchement pour leur couple. Madame quitta alors monsieur et partit vivre avec leurs deux enfants.

Les terreurs nocturnes de Bianca disparurent progressivement au bout de quelques mois; elle devint une jeune fille, commença à s'interesser aux garçons, et réintégra sa chambre et son lit. Mais un nouveau symptôme apparut bientôt: Bianca se coupait régulièrement sur les bras avec une lame de rasoir lorsqu'elle se retrouvait seule dans sa chambre, véritable rituel qu'elle ne pouvait empêcher et qui, disait-elle la soulageait. Elle mettait un point d'honneur à ne jamais montrer ces traces dont elle parlait pourtant volontiers. Jusqu' au jour où sa mère la trouva dans sa chambre en train de se scarifier. Très inquiète, elle en parla au père de Bianca. Monsieur demanda alors seulement à la jeune fille de lui montrer ses cicatrices. Sa réaction angoissa beaucoup Bianca, introduisant dans sa proposition 


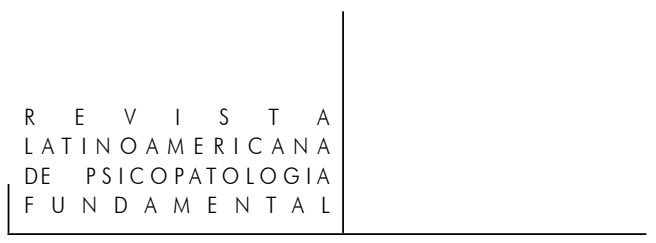

de monstration un rapproché mortifère, la faisant ainsi objet d'une scène primitive scopique. Le symptôme s'accrut.

Par la suite, plusieurs consultations avec la mère de la jeune fille permirent à cette dernière d'évoquer une inquiétante ressemblance entre Bianca et ellemême, lorsqu'elle était adolescente. Madame, bibliothécaire, était l'unique fille d'un père qu'elle n'avait jamais connu - sauf à en porter le nom - et d'une mère, placeuse dans un théâtre, avec laquelle elle avait vécu enfant, mais s'entendait très mal. Elle fit à l'adolescence des tentatives de suicide en se coupant elle aussi, sur les bras, et commença dans le même temps à rechercher son propre père, qu'elle retrouva. Elle ne garda que peu de contact avec ce dernier qui avait refait sa vie et avait d'autres enfants; Bianca ne le rencontra qu'à deux reprises. Madame avait écrit et publié quelques mois auparavant un livre sur cettte histoire - l'histoire de sa famille -, où elle mettait en question une affaire politique assez connue ayant eu lieu en France après la seconde guerre. Bianca faisait le soir des lectures publiques des passages du livre de sa mère, dans des librairies; elle nous disait qu'elle n'avait pas lu ce livre, qu'elle trouvait trop compliqué, et qu'elle lisait à voix haute sans comprendre. Peut-être plus-tard, quand elle serait grande à dix-huit ans, le lirait-elle...

Pendant ce temps, elle continuait inlassablement de se scarifier, toujours au même endroit. C'était comme une écriture sur les bras, une écriture en image, qui ne prenait pas sens. Elle redoubla sa troisième, convaincue de ne pas être faite pour les études, d'“être nulle", et qu'elle serait orientée. Nous lui avons proposé à cette époque de nous apporter un exemplaire du livre de sa mère, afin que nous puissions le lire. Elle manqua quelques unes de ses séances, puis, au bout de quelques semaines, revint avec: elle avait finalement commencé à le lire. Quelques temps après, les scarifications s'arrêterent. La récupération du livre de la mère au lieu des séances, livre qui se promenait, voire qui errait, d'une certaine manière, dans le monde, permit de circonscrire le symptôme de la jeune fille: un certain refus de savoir. ${ }^{6}$ Elle passa en seconde, eut son brevet et poursuivit une scolarité dans laquelle elle semblait s'investir un peu autrement, en commençant à accepter d'apprendre.

6. Ainsi, si le livre de la mère errait dans le monde pour la jeune fille, cette dernière semblait elle aussi jusque là au-dehors, en dehors du lieu du savoir. Pour exemple l'activité principale qui l'occupa cette année là était de s'installer sur le banc d'un square du quartier de la ville où elle habitait, de s'allonger et de "ne penser à rien". Soulignons à propos de l'errance des adolescents la très interessante remarque d'Olivier Douville: "Les adolescents en errance soulèvent une interrogation constante sur notre capacité de créer de l'espace, de présenter des places fiables, des altérités langagières. L'invite à la parole comporte une promesse: celle de 
Autre exemple. Alexia avait 8 ans lorsqu'elle nous a été adressée en thérapie dans le service où nous travaillons. Elle était alors en CE1 au Groupe Scolaire thérapeutique ${ }^{7}$ et en grande difficultés scolaires. Elle ne savait ni lire, ni écrire. H.I.V furent les trois lettres avec lesquelles l'équipe nous parla d'Alexia. Alexia est HIV positive - la maladie a été transmise par sa mère in utéro -, elle est suivie médicalement pour cela et est sous trithérapie. ${ }^{8}$ Monsieur n'a pas contracté la maladie. Née en France de deux parents originaires de Côte d'Ivoire, elle avait été plaçée en foyer à l'âge de 4 ans suite à des maltraitances de sa mère à son égard, mère avec laquelle elle vivait seule. Un signalement des voisins avait conduit à un placement en urgence. Son médecin consultant qui nous l'avait adressé à l'époque hésitait à proposer un espace pour elle-même car elle était "en partance": en attente d'un placement dans une famille d'accueil dans les six mois à venir, et atteinte d'une maladie grave. Avions-nous le temps?

Nous proposons de la recevoir quand-même - on ne sait jamais quand une famille d'accueil va pouvoir être trouvée pour un enfant, cela peut parfois être long. Nous pouvions, après tout, commencer à travailler. Alexia fut non lectrice jusqu'à la fin du primaire. L'institutrice de la Petite école $e^{9}$ disait avoir rarement rencontré une petite fille aussi "butée". Alexia ne voulait rien, mais rien savoir. Il nous apparut très vite au cours des séances non pas qu'elle ne savait pas mais qu'elle refusait plutôt de lire et d'écrire.

Fille d'un père éboueur dans un quartier populaire de Paris, qu'elle voyait à une fois par an lorsqu'il venait lui rendre visite au foyer, et dont elle portait le nom, Alexia ne savait pas grand chose de l'histoire de sa famille. Le service éducatif qui s'occupait d'elle nous avait transmis quelques éléments du dossier. Son père avait d'autres enfants, une famille en Afrique, une femme et plusieurs enfants en

miser, en présence d'un autre, sur les capacités évocatrices et métaphoriques de la parole". Il poursuit: "Le psychanalyste viendrait là faire un peu entame à cette continuité bétonnée et solidifiée qui immerge le sujet dans un temps répétitif, lancinant, où il s'anesthésie". Douville, O. (2007). De l'adolescence errante. Variations sur les non-lieux de nos modernités, p. 58.

7. Institution donc la fonction est celle d'un hôpital de jour avec des instituteurs proposant des temps de soins et des temps scolaires sur place, à temps partiel ou à temps complet.

8. HIV signifie médicalement que la patiente présente un réponse positive aux tests sérologiques HIV. Elle a contracté le virus de l'immunodéficience humaine (VIH), rétrovirus infectant l'homme et responsable du syndrôme d'immunodéficience acquise (SIDA), qui est un état affaibli du système immunitaire le rendant vulnérable à de multiples infections. La patiente suit un traitement sous trithérapie depuis l'âge de 3 ans.

9. Autre nom donné dans le service au Groupe Scolaire Thérapeutique. 


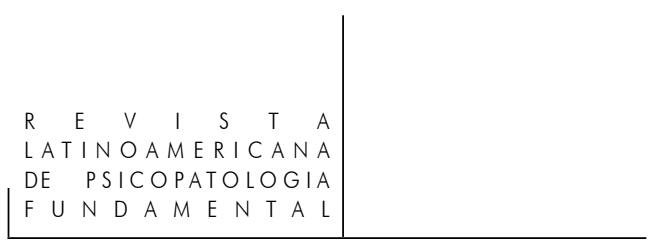

France avec lesquels il vivait - et dont Alexia ne connaissait pas les prénoms. Pendant les premiers mois de séances, Alexia faisait semblant de nous attacher sur une chaise et de nous faire mal, avec des seringues pour prendre le sang. Alexia disait ne pas savoir comment elle avait attrapé cette maladie, seulement qu'elle avait été placée parce que sa mère lui faisait des choses qui faisaient mal. Elle ne lui en voulait pas, et continuait via une médiation à voir sa mère de temps en temps. H.I.V, ces trois lettres du réel prenaient Alexia au corps, empêchant l'accès aux apprentissages.

$\mathrm{Au}$ bout de quelques mois, elle commença en séance un carnet de dessins qu'elle avait appelé "les dessins du peintre $A$ ". Elle plaisantait en disant que son père était un peintre célèbre - il portait en effet le même nom qu'un peintre surréaliste très connu. Ce refus de lire et d'écrire s'inscrivait ainsi dans une difficulté à symboliser la fonction de ce père, qu'elle attendait pourtant avec impatience à chacune de ses visites. Alexia, à 10 ans ne lisait ni n'écrivait toujours pas. Nous décidons alors au bout de deux ans d'entretiens, après en avoir parlé avec elle, d'écrire une lettre au juge et à son service référent de l'Aide Sociale à l'Enfance en insistant sur la nécessité dans ce contexte de trouver une famille d'accueil dans son secteur afin que le travail psychothérapique commencé puisse se poursuivre. Cette lettre eut un effet dans le traitement. Petit à petit, Alexia commença à signer ses dessins, puis à écrire quelques mots sous certains d'entre eux, pour les décrire. Son écriture commençait progressivement à ne plus être seulement une forme. Elle commença à lire quelques mois après, à l'âge de 11 ans.

Un deuxième évènement survint peu de temps après et eut aussi un effet dans le cours de la cure; ce fut l'hospitalisation de sa mère, dont l'état s'était aggravé brutalement. Alexia lui rendit régulièrement visite à l'hôpital, visites au cours desquelles sa mère lui raconta quelques éléments de son histoire et de celle de sa famille, ce qu'elle n'avait jamais fait auparavant. Cette dernière mourut quelques mois plus tard.

Alexia a aujourd'hui seize ans. Elle est en famille d'accueil depuis trois ans, et semble avoir beaucoup investi celle-ci. Elle est en troisième professionnelle et prépare un projet de décoratrice d'intérieur. Elle poursuit encore actuellement ses séances: quelque chose semble finalement s'être mis au savoir au cours de ces huit années.

Oscar

Un dernier exemple clinique, rapidement. Oscar, jeune homme de 25 ans est venu nous voir à notre cabinet, car il souffre d'importantes angoisses qui l'invalident dans sa vie quotidienne, depuis quelques années. Oscar n'a jamais beaucoup travaillé. Vivant seul dans un petit studio depuis quelques mois, il a 
commencé une liaison avec une femme plus âgée, qui le préoccupe beaucoup. Il a essayé de prendre plusieurs traitements (anxiolytiques, puis antidépresseurs) qui n'ont pas marché. Il a été hospitalisé quelques temps auparavant dans une clinique psychiatrique suite à une tentative de suicide mêlant alcool et médicaments, où un diagnostic de psychose maniaco-dépressive a été posé. Après une certaine errance des soins, il rencontra dans une institution de soins ambulatoire où il s'était rendu pour commencer un suivi, un médecin qui l'engagea à commencer une analyse.

Oscar travaille alors depuis quelques mois comme receptionniste dans un hôtel. Il a arrêté ses études en deuxième année d'anglais à l'âge de 20 ans, suite à un conflit avec le nouvel ami de sa mère, qui l'aurait mis dehors de chez eux. Il passe le reste de son temps allongé sur son lit, sans rien faire et s'adonne à des activités auto-érotiques. Il va dans les moments où il va très mal jusqu'à entendre comme des voix qui l'envahissent et lui disent à quel point il serait "nul". Le travail analytique que fit Oscar, allongé, plusieurs fois par semaine pendant sept ans, le conduisit à se défaire d'une identification mortifère à son père, atteint d'une maladie somatique, et ne se soignant pas. Il permit le passage d'un "homme couché" - comme l'appelera Oscar en parlant du mutisme de ce dernier - à un "homme en marche", en déployant sur la châne de ses identifications, son aliénation vis à vis d'un trop de présence maternelle, surmoïque, à laquelle il s'était voué. La bascule de sa position subjective le conduisit à aborder le savoir autrement. Il questionna sa "passion de l'ignorance", ${ }^{10}$ comme l'appelle Lacan passion qui fut mobilisée dans le transfert - et l'associa à la relation qu'il avait à son Autre de l'amour. Il la fit passer du coté de l'envie, d'un véritable appétit de savoir. Il se remit à lire - ce qu'il n'avait plus fait depuis de nombreuses années -, rencontra une jeune femme avec laquelle il s'engagea et reprit des études d'histoire de l'art.

\section{Lectures du désir?}

Un acte du sujet

Ces quelques exemples cliniques nous évoquent une jolie phrase qu'énonçait il y a quelques temps, une de nos connaissances: "tant que je ne voudrais pas

10. Lacan emploie cette expression dans son premier séminaire. Nous le développerons un peu plus avant, p. 13-14. Lacan, J. (1953-54). Séance du 30 juin 1954. Le séminaire. Livre 1. Les écrits techniques de Freud, p. 297-299.

Rev. Latinoam. Psicopat. Fund., São Paulo, v. 13, n. 4, p. 599-616, dezembro 2010 


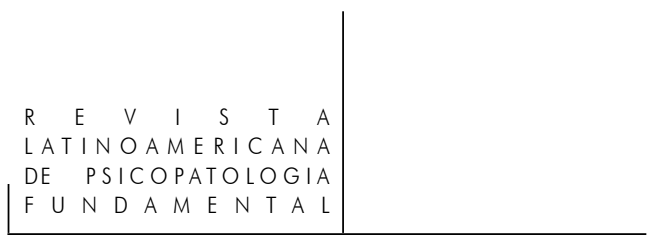

savoir, je ne pourrais pas apprendre!". Lire est, à notre sens, un acte du sujet. Le rapport d'un sujet à la lecture et au savoir pose avant tout la question de sa place d'enfant l'instituant comme sujet de désir et des possibilités qu'il a eu de refouler le savoir inconscient quant à cette place. La clinique psychanalytique notamment des enfants - nous enseigne pour chacun de nos patients, particulièrement, comment autour de sa venue au monde, cela parle de lui. Comme le remarque Catherine Mathelin (2003) "il suffit d'écouter un enfant (...) pour entendre que l'objet de la pulsion épistémophilique porte sur les origines. "Qui m'a fait?" La question des origines, du sexe, de la mort prend les enfants au corps" (p. 136). Ils savent - souligne-t-elle - d' un savoir inconscient, "qui ne se sait pas", comme dit Lacan (1960) dans Subversion du sujet et dialectique du désir, "qui ne comporte pas la moindre connaissance, en ce qu'il est inscrit dans un discours" (p. 803). Ainsi, se nouent, comme Freud déjà le développait, l'envie d'apprendre et la censure qui s'y rapporte. Elle s'origine dans la dialectique pulsionnelle et comme le développe Annie Cordié nous trouvons à sa source tous les objets petit $a$ - oral, anal, scopique, ainsi que la voix (1993, p. 207).

\section{Un déplacement du regard}

Freud, dès ses Trois essais sur la théorie sexuelle en 1905, lie le désir de savoir à la pulsion sexuelle. Il voit dans cette pulsion la sublimation du besoin de maîtrise (analité) et du scopique (désir de voir). Il définit la curiosité intellectuelle comme un destin de la pulsion scopique - inhibée quant au but -, de l'intérêt pour la vision des organes sexuels. Il est nécessaire en effet que le regard se mobilise de ce seul intérêt là pour passer à une forme de sublimation. Il est alors amusant de noter que les difficultés en lecture - affectant la lecture à haute voix et la compréhension du langage écrit - s'appellent en neurologie cécité verbale. En effet, du point de vue de l'organe, la lecture fait appel à une certaine motricité de l'oeil. Et il faut un certain déplacement du regard pour passer du coté de la mise au savoir. C'est donc d'abord une question pulsionnelle, qui concerne, comme le développe Lacan, les orifices du corps.

Lire engage d'abord le corps dans son rapport à la pulsion. La pulsion scopique mais aussi la pulsion orale et la pulsion invoquante et leurs destins y sont impliquées. La parole sort de la bouche pratiquement comme objet même, et c'est de ce lieu "bouche" que va se mettre en mouvement le trajet de ces deux pulsions, orale et invoquante. Ainsi, ce qui prend fonction pour un sujet - pris avant même sa naissance dans un réseau signifiant - c'est ce qui va faire coupure avec ses orifices corporels. Et cette coupure a lieu très tôt dans la vie d'un sujet. Déjà un petit bébé de quelques semaines qui se tient dans les bras de sa jeune maman qui le regarde tendrement, regarde le plus souvent ailleurs, au-delà, c'est un 
phénomène clinique de tous les jours assez étonnant. Jean Bergès (1997) souligne justement que "si la mère tient toutes les fonctions, c'est par son fonctionnement que l'enfant la déborde de toute part" (p. 131). Ainsi l'enfant, par son activité même, son action corporelle et psychique, sa mise en mouvement va au-delà, dépasse, franchit le maternel comme fonction. Et ce franchissement est avant tout permis par ce qu'une mère va dire à son bébé, en tant qu'elle-même est ailleurs, soit désirante du père ou de ce qui en tient lieu. La parole de la mère fait ainsi coupure entre son propre corps et celui du bébé. L'introduction de la parole dans le corps vient entamer, diviser le texte de celui-ci, le ponctuer. Cette parole de la mère, à la fois signifiante et objet même, chute de sa bouche comme orifice..$^{11} \mathrm{De}$ cette coupure à la fois pulsionnelle et signifiante se perd la voix comme objet petit $a .^{12}$ Elle déplace aussi la question scopique entre la mère et l'enfant en permettant que le regard de ce dernier puisse se tourner vers un $x$ où le savoir se loge.

\section{De l'ignorance}

On oublie souvent un point à notre sens important au cours d'une analyse, c'est la fonction de ce que Lacan (1953-54) appelle dès son premier séminaire, "la passion de l'ignorance". L'ignorance, comme l'amour ou la haine est une passion de l'être, à la jonction du réel et du symbolique, et tiendrait comme le cas d'Oscar l'évoque, à la relation à l'Autre de l'amour. Ainsi, écrit Lacan, "le sujet qui vient en analyse se met dans la position de celui qui ignore". Ignorer, non pas au sens péjoratif mais au sens de ne pas connaître, ne pas savoir. Alors, comment mettre en question - en mouvement - dans le travail analytique via le transfert cette "ignorance", afin si c'est possible, de la faire passer du côté d'un travail de pensée, d'une mise au savoir, c'est à dire à accepter - ou à pouvoir être à nouveau capable - de connaître, d'apprendre? Jeanne Lafont (2006) emploie à ce propos la jolie expression d'une "torsion du regard" au cours de la cure. Comme elle l'a développé dans une intervention au Centre référent des apprentissages, à l'hôpital Sainte-Anne: "le psychanalyste reste supposé savoir. Il faut qu'il en sache un bout pour écouter. Le psychanalysant, l'enfant, ou l'adolescent est au travail de l'inconscient. Il peut retrouver le lieu d'un choix de l'ignorance, choix inconscient qui l'aliène".

11. Bergès souligne ainsi: "elle (la parole) sort de sa bouche, de sa bouche qui remue (...) placée dans le regard de l'enfant exactement de la même façon que lorsqu'elle lui donne à manger". Ibid. p. 132.

12. Lacan développe ce point dans Subversion du sujet et dialectique du désir. Lacan, J. (1960). Subversion du sujet et dialectique du désir dans l'inconscient freudien. In: Ecrits, p. 793-828. 


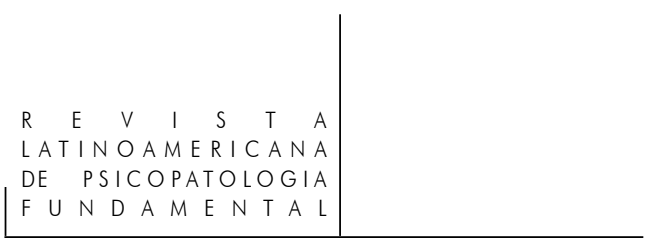

\section{Le temps de l'intervalle ${ }^{13}$}

Le langage est comme un jeu de construction. Si les mots offrent parfois le même son à l'oreille, ils possèdent pourtant plusieurs sens. Les mots lus dans une phrase vont s'articuler avec des tempos, des temps d'arrêts, d'où vont se perdre des sons mais aussi quelques lettres. Irrémédiablement perdues, presque en même temps qu'elles sont constituées, ${ }^{14}$ c'est leur fonction de perte qui permet d'accéder au sens d'un texte, à l'équivoque ${ }^{15}$ ou aux jeux de mots.

Cette perte est tout d'abord permise par la construction de la ponctuation dans un texte. De même qu' on ne peut pas tout dire, que la parole est mi-dire, on ne peut pas non plus tout écrire. La lecture résulte de cette perte là de ce passage de la vérité au savoir. Crochets, points d'exclamations, guillemets, points d'interrogations, parenthèses, points virgules, tirets, virgules, points de suspensions se croisent et marquent un certain rapport au temps dans un texte, soulignent ses divisions et ses nuances, ses intervalles. La ponctuation permet de comprendre un texte - comme à la lecture du texte inconscient au cours d'une cure analytique. Elle indique dans une phrase, comme dans un morceau de musique, les repos, les silences. Elle scande le texte. "Temps de l'intervalle", comme l'appelle Pierre Fédida (1978), elle permet l'espace psychique de la métaphore.

Ne pas accéder à la lecture, n'est-ce pas aussi, d'une certaine manière, refuser ces temps, ces silences qui font césure, division pour un sujet?

\section{Le réel de la lettre}

Les signifiants - phonèmes, lettres, caractères -, supports d'un sens, sont énoncés et articulés par la voix d'un sujet. La parole, selon le sens à donner à ceux-ci, en module ses changements de tons, d'accents, d'intensités (Lacan, 1957). Signifiants, voix, sont émis sans pourtant se rencontrer tout à fait lorsque la mère ou l'enfant communiquent, ce qui va produire un effet de perte.

Jean Bergès (1997, p. 316) développe dans ses travaux pourquoi cet effet de perte est justement en question dans l'acte de lire, comme dans celui d'écrire d'ailleurs, mais différemment . On écrit sur le corps de la mère - pour reprendre son expression souvent employée - en tant que celui-ci est troué et que dans son

13. Nous empruntons cette expression à Pierre Fédida. Fédida, P. (1978). L'absence, p. 221.

14. On peut souligner ici le point commun entre ces lettres perdues et la chose, das Ding.

15. Le terme "équivoque" vient du latin équivocus qui signifie "à double sens" et de vox la "parole", la "voix". Article "équivoque". In: Dictionnaire Le petit Robert, p. 935. 
rapport à l'enfant, il y a pour ce dernier un effet de coupure. Dans la lecture, nous l'avons évoqué, il est nécessaire de perdre des lettres, soit que le texte soit un peu manquant pour accéder au sens. Dans l'écriture les choses sont un peu différentes. Le cas d'Alexia montre bien combien écrire est aussi un acte, celui de cesser tout d'abord d'être seulement un dessin, soit quelque chose qui a affaire avec le corps dans sa dimension réelle et imaginaire. Ecrire est un acte, à distinguer de l'acte graphique, qui concerne d'une certaine manière la forme de l'écriture. De plus, quand le sujet écrit, il ne parle pas, et perd aussi quelque chose du côté de la voix (p. 324). Consonnes, voyelles, quant-à-elles, n'y doivent pas manquer, pas plus que les accents ou les points sur les $\mathrm{i}$, afin de respecter les règles de la conjugaison, de la grammaire - bref, comme le poète Gainsbourg (1961) l'évoque dans sa chanson, de l'orthographe.

\section{L'acte analytique}

Les formations de l'inconscient se manifestent dans la parole ou dans les actes sous la forme d'une écriture chiffrée - rêves, symptômes, lapsus, actes manqués, mots d'esprit. L'inconscient se donne à lire et son texte à déchiffrer; l'écriture de ce dernier est un rébus, au sens d'un jeu de mots, voire de lettres. Lettre en plus ou en moins, il joue sur l'orthographe différente des mots ayant un même son: l'homophonie. Comme dans la poésie, les mots pris comme des choses valent par leurs rapports et leurs analogies. Les éléments de la chaîne signifiante inconsciente se manifestent dans la parole sur le mode de la lettre, en tant que signe d'un désir interdit. L'inconscient tente ainsi de déjouer la censure en rapport avec ce que nous avons dû refouler pour advenir comme sujet, et renoncer à être le seul objet du désir de la mère. Le sujet de l'inconscient pourtant sans voix - se fait entendre.

L'acte analytique vient ainsi inscrire une ponctuation. Le maniement par l'analyste d'une certaine équivoque dans le langage - signifiante, grammaticale, homophonique - permet de faire apparaître de nouveaux sens. L' humour, comme la poésie, en sont une version. L'analyste - de par son désir - a finalement, dans notre civilisation actuelle, et face à ces "nouveaux symptômes" qu'expriment les sujets, cette fonction d'un poète. Le désir de l'analyste insiste Alain Didier-Weill (2003), "causé par ces objets “ $a$ " que sont la voix et le regard: (...) l'un par sa façon de faire entendre l'inouï et l'autre par sa façon de donner à voir l'invisible est énigmatiquement de structure poétique" (p. 122). Il l'amène à entendre un certain malaise dans la civilisation et à s'en occuper. 


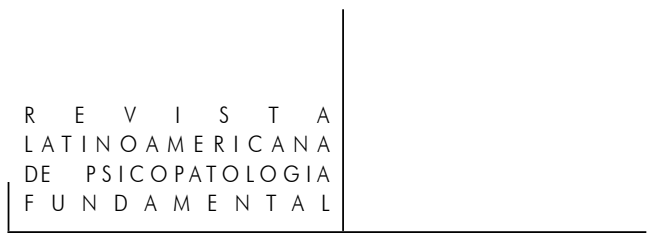

\section{Références}

BERGÈs, J. (1997). Le corps dans la neurologie et dans la psychanalyse. Paris: Eres, 2005.

Bergès, J.; Bergès-Bounes, M.; Jean-Calmettes, S. (27/10/2002). "Que nous apprennent les enfants qui n'apprennent pas?". Conférence à l'Association Lacanienne Internationale, publiée dans l'éditorial du Journal Français de Psychiatrie, n. 15.

Berube, L. Traité de terminologie de neuropsychologie et de neurologie du comportement. Paris: Recherche et réd., 1991.

Cordie, A. Les cancres n'existent pas. Paris: Seuil, 1993.

Didier-WeILl, A. Lila ou la lumière de Vermeer. Paris: Denoël, 2003.

Douville, O. De l'adolescence errante. Variations sur les non-lieux de nos modernités. Paris: Éditions Pleins Feux, 2007.

FÉDIDA, P. L’absence. Paris: Gallimard, 1978.

Freud, S. (1905). Trois essais sur la théorie sexuelle. Paris: Gallimard, Folio Essais, 1987. . (1929). Le malaise dans la culture. Paris: PUF Quadrige, 2002.

GaInsBourg, S. En relisant ta lettre. In: Mon propre rôle. Textes. 1958-1975. Paris: Denoël, 1961.

LaCAN, J. (1953-54). Séance du 30 juin 1954. Le séminaire. Livre 1. Les écrits techniques de Freud. Paris: Seuil, 1975. p. 297-299. . (1957). L'instance de la lettre dans l'inconscient depuis Freud. In: Écrits. Paris: Seuil, 1966. p. 493-530. . (1960). Subversion du sujet et dialectique du désir. In: Écrits. Paris: Seuil, 1966. p. 793-828. . (1972-73). Le séminaire. Livre XX. Encore. Paris: Seuil, 1975.

LAFOnT, J. (7 mars 2006). Intervention inédite au Séminaire de l'Unité de Psychopathologie de l'Enfant et de l'Adolescent. Centre référent pour le diagnostic et la prise en charge médicale des troubles spécifiques d'apprentissage du langage oral et écrit. Service de Psychologie et Psychiatrie de l'Enfant et de l'Adolescent, 6ème intersecteur, Centre-Hospitalier Sainte-Anne, Paris.

. Les dessins des enfants qui commencent à parler. Paris: Efeditions, 2006.

Mathelin, C. Ce que j'entends, je ne peux pas l'écrire. In: Bergès, J. et M. Calmettes-Jean, S. Que nous apprennent les enfants qui n'apprennent pas? Ramonville Saint-Agne: Eres, 2003. 
(Alexia hoje: algumas hipóteses sobre um sintoma do nosso tempo)

A dificuldade de ler - ou mesmo escrever - de um sujeito é um sintoma atual da nossa civilização. Vários exemplos clínicos questionam a relação entre o aprendizado e o saber inconsciente. A origem da vontade de aprender é abordada por Freud nas pulsões, assim como a necessidade de perda do objeto a, considerando-se particularmente o olhar e a voz. No âmbito do real, a função das letras que se perdem é também levada em consideração. A direção da cura e a relevância da sua temporalidade são abordadas objetivando uma leitura do inconsciente como um jogo de palavras e de letras.

Palavras-chave: Alexia, saber, corpo, pulsão, objeto $a$

(Alexia today: hypotheses about a symptom for our times)

A subject's difficulty to read - or even write - is a current-day symptom of our civilization. Several clinical examples question here the relationship between learning and unconscious knowledge for a subject. The origin of the will to learn, related to the drives, was taken up by Freud. This has also been discussed in relation to the loss of object a (especially in terms of the gaze and the voice). In the sphere of the real, the function of letters that are lost is also examined. The direction of the treatment and the importance of its temporality are thus approached in order to provide a reading of the unconscious as an interplay of words and letters.

Key words: Alexia, knowledge, body, drive, object $a$

(La alexia hoy. Algunas hipótesis sobre un síntoma de nuestro tiempo)

La dificultad de leer - o mismo escribir - de un sujeto es un síntoma actual de nuestra civilización. Múltiples ejemplos clínicos interrogan la relación entre aprendizaje y saber inconsciente para un sujeto. El origen del deseo de aprender es ligado por Freud a las pulsiones, así como también a la necesaria función de la perdida de los objetos a (particularmente la mirada y la voz). Del lado de lo real, la función de las letras que se pierden es llevada en consideración. La dirección de la cura y la relevancia de su temporalidad es abordada con miras a una lectura del inconsciente como un juego de palabras y de letras.

Palabras claves: Saber, cuerpo, pulsión, objecto $a$ 
R E V I S T A

LATINOAMERICANA

DE PSICOPATOLOGIA

$F \cup N D A M E N$ T A L

Citação/Citation: Pellion, T. L'alexie aujourd'hui. Quelques hypothèses sur un symptôme de notre temps. Revista Latinoamericana de Psicopatologia Fundamental, São Paulo, v. 13, n. 4, p. 600-617, dez. 2010.

Editor do artigo/Editor: Prof. Dr. Manoel Tosta Berlinck

Recebido/Received: 21.8.2009/ 8.21.2009 Aceito/Accepted: 10.11.2009/ 11.10.2009

Copyright: () 2009 Associação Universitária de Pesquisa em Psicopatologia Fundamental/ University Association for Research in Fundamental Psychopathology. Este é um artigo de livre acesso, que permite uso irrestrito, distribuição e reprodução em qualquer meio, desde que $\mathrm{o}$ autor e a fonte sejam citados/This is an open-access article, which permits unrestricted use, distribution, and reproduction in any medium, provided the original author and source are credited.

Financiamento/Funding: A autora declara não ter sido financiada ou apoiada/The author has no support or funding to report.

Conflito de interesses/Conflict of interest: A autora declara que não há conflito de interesses/The author declares that has no conflict of interest.

Tatiana Pellion

Docteur en sciences humaines cliniques (Psychopathologie Fondamentale et Psychanalyse). Psychologue clinicienne, 6ème intersecteur de Psychologie et Psychiatrie de l'Enfant et de l'Adolescent, 14 ème arrondissement de Paris, Centre Hospitalier Sainte-Anne. Psychanalyste; Chargée de cours à l'université Paris VII-Denis-Diderot, et à l'université Paris XIII-Villetaneuse, chercheur associée au Centre de Recherche Psychanalyse et Médecine de l'Université Paris VII-Denis-Diderot, adhérente à Espace Analytique.

49, boulevard de Port-Royal

75013 Paris, France

e-mail: tatiana.tresmontant@wanadoo.fr 